\section{Current Management of Renal Trauma}

\author{
Hannah Wells and Bhaskar K Somani*
}

Department of Neonatal surgery, University Hospital Southampton NHS Foundation Trust, Southampton, UK

\begin{abstract}
Traumatic renal injuries remain a potentially life threatening presentation following blunt and penetrating abdominal trauma. It is therefore important that these cases are treated urgently and efficiently to prevent renal damage and the risk of associated bleeding. We present an update on management of patients with renal trauma.
\end{abstract}

Keywords: Embolization; Kidney; Management; Nephrectomy; Trauma

\section{Introduction}

Unintentional injuries and violence are a major public health issue causing the death of more than nine people every minute worldwide [1]. It is estimated that 2 million people in the USA need hospital admissions for trauma related injuries [2]. While the abdomen is the third most common site of injury, kidney is the most commonly injured genitourinary organ with renal trauma occurring in up to $5 \%$ of cases [3].

Renal trauma can be sub-classified as blunt or penetrating injuries. Blunt renal trauma is more common, comprising $60-95 \%$ of renal trauma cases depending on whether it has happened in an urban or rural location [3]. Blunt renal injuries are caused by motor vehicle collisions in nearly half of the cases and include pedestrian injuries, with the remainder from sport related injuries and assault. Gunshot and stab wounds account for most common penetrating injuries and tend to be more severe and potentially less predictable in its outcome.

Renal trauma can be classified according to the 1989 American Association for the Surgery of Trauma (AAST) grading system (Table 1). This was revised in 2011 to report grade $\mathrm{V}$ injuries as purely vascular/pedicle injury [4] but some studies still use the original AAST grading system.

*Corresponding author: Bhaskar $\mathrm{K}$ Somani, Department of Urology, University Hospital Southampton NHS Foundation Trust, Southampton, UK Tel: +44 2381205272; E-mail: bhaskarsomani@yahoo.com

Citation: Wells H, Somani BK (2015) Current Management of Renal Trauma. J Emerg Med Trauma Surg Care 2: 009

Received: May 14, 2015; Accepted: August 10, 2015; Published: August 24 2015

\begin{tabular}{|c|c|l|}
\hline Grade & Type of Injury & \multicolumn{1}{|c|}{ Description } \\
\hline I & $\begin{array}{c}\text { Contusion } \\
\text { Haematoma }\end{array}$ & $\begin{array}{l}\text { Microscopic or gross haematuria with normal urologic } \\
\text { findings } \\
\text { Non-expanding subcapsular haematoma with no } \\
\text { laceration }\end{array}$ \\
\hline II & $\begin{array}{c}\text { Haematoma } \\
\text { Laceration }\end{array}$ & $\begin{array}{l}\text { Non-expanding perinephric haematoma confined to } \\
\text { retroperitoneum } \\
\text { Superficial cortical laceration }(<1 \mathrm{~cm}) \text { without collecting } \\
\text { system injury }\end{array}$ \\
\hline III & Laceration & $\begin{array}{l}\text { Renal laceration >1cm in depth without collecting } \\
\text { system injury }\end{array}$ \\
\hline Laceration & $\begin{array}{l}\text { Renal laceration extending through renal cortex, medulla } \\
\text { and collecting system } \\
\text { Injuries involving main renal artery/vein with contained } \\
\text { haematoma, segmental infarctions without associated } \\
\text { lacerations }\end{array}$ \\
\hline V & $\begin{array}{c}\text { Laceration } \\
\text { Vascular injury }\end{array}$ & $\begin{array}{l}\text { Shattered kidney, ureteropelvic junction avulsions } \\
\text { Complete laceration (avulsion) or thrombosis of main } \\
\text { renal artery/vein that devascularises the kidney }\end{array}$ \\
\hline
\end{tabular}

Table 1: AAST classification of renal injuries [3]

Management of renal injuries often directly relates to the grade of injury at diagnosis. While the management of low-grade injuries is more straightforward, there is uncertainty over the management of higher-grade injuries, particularly regarding the role of conservative management. Over the last decade there has been a trend towards conservative management of high-grade renal injuries, which helps in preserving the renal function. Here we lay out an updated approach to management of patients presenting with renal trauma based on up-to-date evidence.

\section{Immediate Management}

On presentation to the emergency department, trauma patients are assessed and resuscitated following the principles of Advanced Trauma Life Support (ATLS). When obtaining the history, possible indicators of renal injury in blunt trauma include rapid deceleration or direct blow to the flank [5]. Patient history is important as pre-existing Pelvo-Ureteric Junction (PUJ) obstruction, cysts and tumours can complicate a perceived minor injury. On examination, after a blunt injury, location of bruising can indicate underlying renal trauma while location of penetrating wounds is important to note to determine if a renal injury has occurred. Continuous haemodynamic monitoring in the emergency department contributes to decision making regarding investigation and management in these patients.

\section{Investigation}

When a trauma patient arrives in the emergency department, a rigorous systematic evaluation is performed including sending a full set of blood tests. When renal trauma is suspected, some of these laboratory tests are particularly important including full blood count and serum creatinine measurement to aid baseline assessment and follow up of their renal function. A urine sample should also be collected to look for any gross or microscopic haematuria. In unstable patients, this is usually from a catheter specimen, which is also used for monitoring the urine output. This should be inspected for visible haematuria and if clear on inspection, urinalysis should be performed 
to assess for any microscopic haematuria. However, in up to $9 \%$ of patients significant renal injuries happen without haematuria [6], for instance in cases of disruption of the PUJ and pedicle injuries.

Further investigation often depends on the haemodynamic stability of the patient. If the patient is unstable despite adequate resuscitation, there is often a need for emergency exploratory laparotomy and the patient is transferred to the operating theatre. In this situation, it is possible to perform an on-table Intravenous Pyelogram (IVP). Most renal trauma guidelines and review articles indicate the need for on-table IVP to assess the contralateral kidney when making a decision regarding renal exploration, especially when there is a relative indication, although in reality, this is rarely necessary. IVP has largely been superceded by contrast CT scan as the investigation of choice for diagnosing renal injuries.

CT scanning is more sensitive and specific than IVP and ultrasound scanning. It provides greater anatomical detail and associated injuries to any intra-abdominal organs. For renal injury, a scan should also be performed after injection of intravenous contrast to delineate the collecting system (delayed phase). CT scanning is the only imaging modality that can provide information required to grade renal injuries as part of the AAST renal injury grading system. For this purpose, there needs to be an arterial phase, a venous phase and a delayed excretory phase to assess for all pedicle injuries [7]. As per EAU guidelines, contrast CT scan is the gold standard for diagnosis and staging of renal injuries in haemodynamically stable patients [3].

\section{Grading}

Renal injuries are graded as part of the AAST renal injury grading system (Table 1). This includes extent of haematoma, depth of laceration, involvement of the collecting system and severity of vascular injury. Recent articles have proposed revisions, including moving all collecting system injuries to be classed as grade IV injury and including only hilar injuries in grade V [4]. The same group have suggested addition of segmental vascular injury and complete PUJ obstruction to grade IV and main renal artery/vein thrombosis to grade $\mathrm{V}$ [4]. This reflects the severity of completely occlusive thrombus and the need to consider a two hour time limit for revascularisation [7].

Outside of the AAST renal injury grading system, there are other related pathologies that can influence management decisions either in the immediate setting or later in the admission. Active contrast extravasation from branching renal arteries (i.e., active small artery bleeding, arterial pseudoaneurysms and arterio-venous fistula are all pathologies related to renal trauma that will be picked up on the trauma CT, require active management but are not yet included in the grading system [7].

Alongside the grading system, there has been research into factors that may predict need for intervention [8]. Certain high risk criteria seem to be apparent in renal trauma and it seems that when two or more of these are present, the patient is likely to require intervention (either surgery or embolisation) to manage bleeding [9]. One group has suggested that perirenal haematoma size $(>3.5 \mathrm{~cm})$, active intravascular contrast extravasation and medial laceration site correlate with need for urgent intervention [8]. They have concluded that a Grade IVb should be added to the existing guidelines which would include Grade IV injuries with two or more of these CT findings.

\section{Open Intervention}

Open surgery and exploration rates vary widely although there has been a trend towards fewer open surgical interventions for renal trauma with growing evidence for conservative 'wait and see' management or minimally invasive techniques [7]. An updated management pathway for blunt and penetrating injuries is summarised in figure 1 . Nephrectomy rates up to $22 \%$ have been quoted [10] but this is for grade $\mathrm{V}$ injuries and the need for intervention seems to increase with increasing injury grade [7].

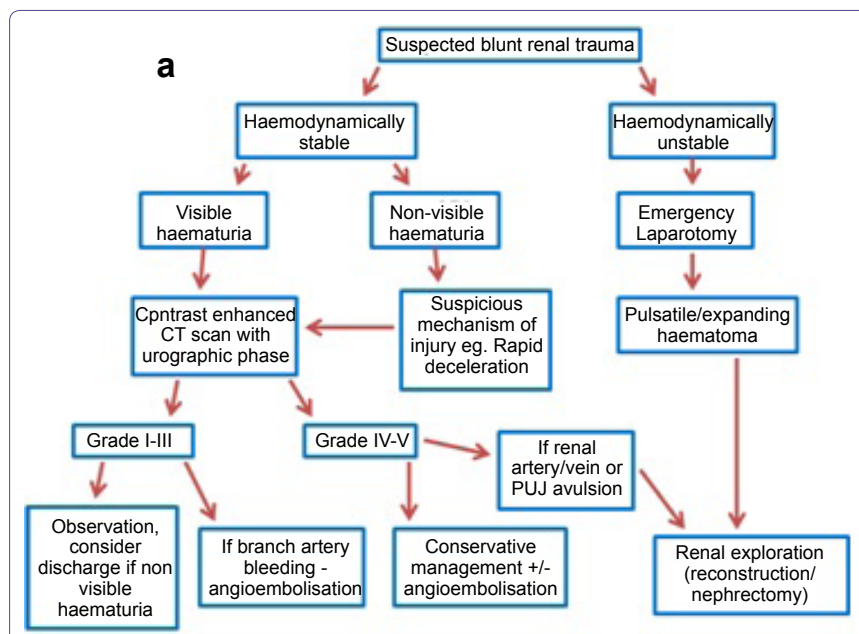

Figure 1a: Management pathway for renal trauma. Suspected blunt renal trauma. (Non-visible haematuria refers to any dipstick positive reaction for blood on urine testing).

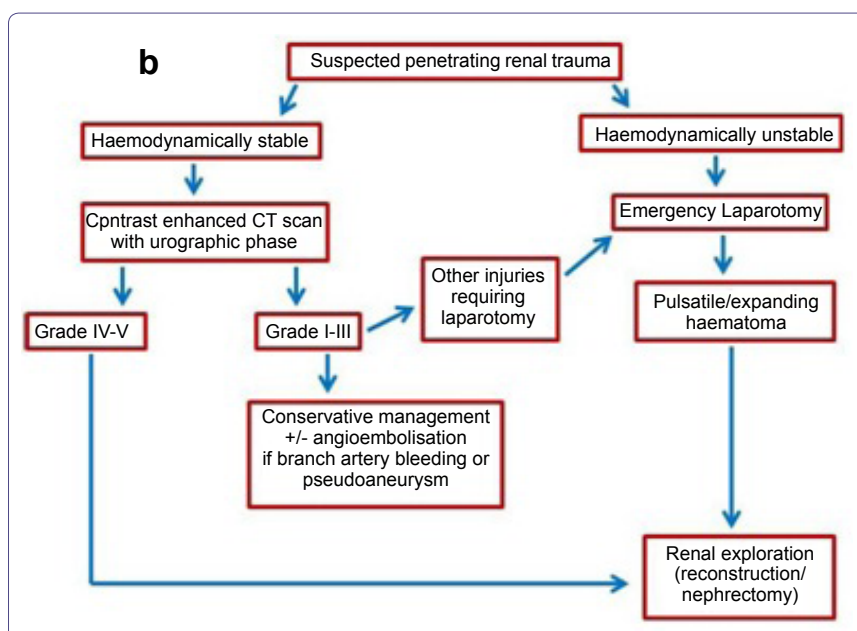

Figure 1b: Management pathway for renal trauma. Suspected penetrating renal trauma $[3,7]$. (Non-visible haematuria refers to any dipstick positive reaction for blood on urine testing).

Haemodynamic instability despite adequate resuscitation measures remains an absolute indication for surgical exploration of trauma patients. This can often require a multi-specialty team approach as these patients will not be able to undergo imaging to diagnose the source of bleeding. In more than $80 \%$ of patients who had surgical exploration where a renal injury was identified, another severe, non-urological injury mandated immediate exploration [11]. Pulsatile or expanding perinephric haematoma identified at laparotomy is also quoted as an absolute indication for renal exploration by most centres. When a stable renal haematoma is 
identified at exploratory laparotomy, this should be left alone to avoid disrupting Gerota's fascia and releasing the tamponade effect. In such cases exploration could potentially lead to nephrectomy, which could otherwise have been avoided [12].

Renal reconstruction can be attempted during open exploration once haemorrhage is controlled. Depending on the underlying injury, various reconstruction techniques have been used including primary renorrhaphy, omental flap, partial nephrectomy and vascular repair [13]. The primary aims during exploration and reconstruction are debridement of non-viable tissue, ligation of bleeding vessels and water tight collecting system repair. Different centres report variable rates of use of reconstructive surgical options but there is evidence of use of renorraphy (direct suture repair of the kidney) and partial nephrectomy (removal of the devitalised area of kidney with preservation of the remaining portion incorporating arterial and venous supply plus ureteric drainage) across all injury grades where appropriate [14].

In the past, with blunt trauma, high grade injuries (IV and V) on imaging would have been an indication for operative intervention. Over time, however, particularly with the advent of regional trauma centres, more of these patients have a trial of conservative management where appropriate. In fact, in a systematic review it was reported that $82.9 \%$ of Grade III-V injuries were managed conservatively [15]. In the same study, Grade $\mathrm{V}$ injury was, however, noted as a predictor for need for surgical intervention alongside platelet transfusion during resuscitation (possibly reflecting the use of platelets when higher volumes of packed red cells have been transfused). This is supported by an Indian study that looked at a series of patients and found that Grade V injuries and more packed red cell transfusions during resuscitation predicted the need for emergency operative intervention [16]. Following blunt trauma, repair of grade $\mathrm{V}$ vascular injuries is seldom possible and should only be attempted if it is a solitary kidney. With these injuries, the outcome of nephrectomy versus repair is comparable in the long term. Grade IV and V injuries should be managed with care with close attention to haemodynamic stability, mechanism of injury and associated injuries to determine the need for intervention [13].

Penetrating traumatic renal injuries are often more commonly explored in the initial phase than blunt renal injuries [17]. In addition, penetrating injuries tend to end up in the operating room sooner than blunt injuries (30 minutes vs 120 minutes) [14]. Patients undergoing exploratory laparotomy were more likely to end up having a nephrectomy than renal trauma patients in general [14]. This may reflect the severity of injury in patients that require exploratory laparotomy but also supports the use of a trial of conservative management to aid renal parenchymal preservation where appropriate. There is also a higher nephrectomy rate among patients with penetrating renal injuries. As such, again with the development of high quality trauma services lead by multidisciplinary trauma teams at trauma centres, there has been a trend towards trial of conservative management even in patients with penetrating injuries. Some high volume studies have shown that almost $50 \%$ cases with penetrating trauma who remain stable can be managed conservatively with acceptable outcomes [12].

Minimally Invasive Intervention: Angioembolisation

Non-operative management is being increasingly used in many benign and malignant conditions with safe outcomes [18]. In the context of renal trauma, this has coincided with a gradual move away from open intervention based solely on injury grade. This has been boosted by improvements in imaging and the advent of interventional radiology [19]. Although angiography is limited as a sole investigation modality in trauma, it can be useful in blunt and penetrating injuries when there may be a need for embolisation for control of bleeding. In a stable patient with persistent bleeding, angiography can allow selective arterial embolisation [5,7]. This can prevent the need for surgical exploration [20], particularly in sole renal injuries. The selective technique also enables potential preservation of renal parenchyma without procedure related decline in renal function [21].

The use of selective angioembolisation was introduced in the early 1970s for management of acute renal parenchymal and vascular trauma [22]. Initially, this was reported as more successful in lower grade injuries. A recent survey, however, has shown that both urologists and interventional radiologists view embolisation as an option in Grade IV and V injuries [23]. There was particular support for its use in blunt injuries from both groups, while urologists tended to view it less favourably than radiologists when managing penetrating injuries. Use of embolisation as a first line treatment is mostly for active arterial bleeding (particularly branching renal arteries), arteriovenous fistula or pseudoaneurysm [7]. It has also been used as a second line management in patients with declining haemodynamic status after failed conservative management or active bleeding on repeat CT scanning. Success rates of up to $92 \%$ have been reported in a national series with an $88 \%$ success rate in high grade renal injuries [24], although some patients required more than one intervention. The use of angioembolisation and its success is dependent on the availability, knowledge and skills of an interventional radiologist [23].

\section{'Wait and See' Conservative Management}

Increasing numbers of renal trauma patients, even those with high-grade injuries, are now managed with a trial of conservative or 'wait and see' management [7]. In fact, with the advent of specialised trauma centres, conservative management was more common at these regional centres despite patients having more severe renal injuries [25]. Once the patient is clinically stable the primary goals are preservation of renal parenchyma (and therefore function), and minimisation of morbidity [15]. Patients with Grade I renal injuries and without gross haematuria can be safely discharged without any need for further imaging [13].

Patients with grades II and III renal injuries should be observed for the first 24 hours on bed rest [11]. It is suspected that the highest risk of bleeding occurs during the first 24 hours so monitoring and bed rest is often recommended until gross haematuria has resolved. Low grade fevers $(<38.5)$ are often seen with haematomas and evidence from literature in children suggests that low grade fever alone need not prompt intervention [13].

Non-operative management can also be recommended for penetrating injuries in haemodynamically stable patients when full imaging has been obtained [3]. Low-velocity gunshot wounds and minor stab wounds can be managed conservatively with good outcomes. In contrast, high velocity gunshot wounds cause more extensive damage and often require nephrectomy. When using a trial of conservative management in stab wounds, it is important to bear in mind that injuries $\geq$ grade III can behave unpredictably and often require delayed intervention.

Repeat imaging should be performed to investigate fever, uncontrolled pain and unexplained reduction in haemoglobin. In 
stable patients with grade I-IV renal injuries, however, there is no need for routine repeat CT scanning [26]. Now that it has been shown that repeat imaging is only necessary in cases where there is deterioration in the clinical condition, patients are often discharged early [12]. In fact, renal trauma patients have been safely discharged from trauma centres after less than two days [12], but prospective studies and robust evidence about movement through the trauma pathway are still awaited.

Minimally invasive approaches can be useful in the primary management of renal trauma patients and in management of its complications. Management of urine extravasation presents an area of debate $[10,12]$. In a more recent review, urinoma rate was quoted as up to $7 \%$ on trauma CT [27]. There is debate between monitoring versus early ureteric stent insertion, particularly in patients with blunt injuries. In penetrating injuries, patients may have had exploratory laparotomy with identification and management of urine leak. Close monitoring of these patients has historically involved bed rest, hydration, analgesia and antibiotics. However, in one observational study prophylactic antibiotics were not routinely used but given after collection of culture samples in patients with a fever $>38.5^{\circ} \mathrm{C}$ [28]. In the same study, the patients suspected of sepsis which did not respond to antibiotics were more likely to need stent placement. In one review, $17 \%$ of patients who underwent a trial of conservative management developed subsequent urinoma and these were successfully managed by percutaneous drainage or ureteric stent placement [15]. It is now recognised that ureteric stenting is preferable to percutaneous drainage [12] but it seems that in the absence of sepsis, intervention is only absolutely indicated for ongoing pain from clot colic and ureteral obstruction [10].

Serum creatinine measurement is also important in these patients. While not an immediately life threatening issue compared to active bleeding, Acute Kidney Injury (AKI) can increase lifelong morbidity and mortality and is a severe complication following trauma that should be considered, AKI can influence further investigation (contrast vs. non contrast) and management decisions [21]. Post-traumatic AKI can be caused by multiple factors including the renal injury, haemodynamic instability, rhabdomyolysis and contrast medium. Although many trauma patients are young with minimal co-morbidities, the previous history of the patient can also contribute. Up to $62 \%$ of trauma patients can show an increase in serum creatinine from admission [21] but it appears that most of these return to normal prior to discharge. The immediate investigation and management of these patients is still based on clinical signs and often the trauma CT has been done before renal function results are available as it remains the gold standard diagnostic tool. Later management, however, should consider recovery of renal function. The advent of superselective angioembolisation has provided an effective treatment option without deleterious effects on renal function [21].

\section{Follow Up}

It is recommended that first follow-up be scheduled for three months following hospitalisation [3]. Ideal follow up should involve physical examination, urinalysis, blood pressure, renal function blood tests with individualised imaging. In a paediatric trauma paper, post-traumatic CT was routinely scheduled and performed three months following the injury in patients with high grade renal injury [11]. Nuclear scans are useful for tracking recovery, particularly following renal reconstruction. Assessment of residual renal function with Dimercapto-Succinic Acid Renal Scintigraphy (DMSA) is inconsistent but has been performed by some centres for injuries of grade III or above. One study looked at these results but the scans were performed between 1 and 818 days post injury [29]. It is likely that early DMSA scanning offers no long term information but it has been proposed that renal function six months post injury is not significantly different to renal function on DMSA scanning 8 days post injury [29]. Another study showed that $65 \%$ of patients with urine leak at time of diagnosis had follow up DMSA at 4-6 months [28]. In these cases, operative management and $>25 \%$ devitalised parenchyma at diagnosis independently predicted long term renal function.

Follow up information on renal trauma patients is usually limited. Few papers have any long term outcome data. This is likely a reflection that the patients often reside many miles from the trauma centre where they were managed. It could also be related to the patient demographic being younger, active subjects.

\section{Conclusion}

In all trauma patients, if they remain unstable despite resuscitation, laparotomy remains the first line investigation and management strategy. Absolute indications for renal exploration are expanding/pulsatile peri-renal haematoma. For patients that are stable, CT scanning is the gold standard mode of investigation for grading renal injuries.

Subsequent management can then be based upon the clinical status (Figure 1) in both blunt and penetrating injuries and often begins with a 'wait and see' strategy. Follow-up or 'delayed' CT scans are no longer recommended unless there is deterioration in the clinical picture. Following discharge from the hospital, monitoring of blood pressure, renal function blood tests and individualised nucleotide imaging is necessary for high-grade injuries.

\section{References}

1. WHO (2010) Injuries and Violence: The Facts. Geneva, Switzerland.

2. Guillon F (2011) Epidemiology of Abdominal Trauma. In: Guillon F (ed.). CT of the Acute Abdomen, ( $1^{\text {st }}$ edn). Springer, Heidelberg, Germany. Pg: 15-27.

3. Summerton DJ, Djakovic N, Kitrey ND, Kuehhas FE, Lumen N, et al. (2014) Guidelines on Urological Trauma. European Association of Urology.

4. Buckley JC, McAninch JW (2011) Revision of current American Association for the Surgery of Trauma Renal Injury grading system. J Trauma 70: 35-37.

5. Alsikafi NF, Rosenstein DI (2006) Staging, evaluation, and nonoperative management of renal injuries. Urol Clin North Am 33: 13-19.

6. Eastham JA, Wilson TG, Ahlering TE (1992) Radiographic evaluation of adult patients with blunt renal trauma. J Urol 148: 266-267.

7. Bonatti M, Lombardo F, Vezzali N, Zamboni G, Ferro F, et al. (2015) MDCT of blunt renal trauma: imaging findings and therapeutic implications. Insights Imaging 6: 261-272.

8. Dugi DD 3rd, Morey AF, Gupta A, Nuss GR, Sheu GL, et al. (2010) American Association for the Surgery of Trauma grade 4 renal injury substratification into grades 4a (low risk) and 4b (high risk). J Urol 183: 592-597.

9. Figler BD, Maegler BS, Voelzke B, Smith T, Wessells H (2013) External Validation of a Substratification of the American Association for the Surgery of Trauma Renal Injury scale for Grade 4 injuries. J Am Coll Surg 217: 924-928.

10. Breyer BN, Kovell C, McGeady JB, Tasian GE (2014) Renal Trauma. AUA Update Series 33: 1-12.

11. Buckley JC, McAninch JW (2004) Pediatric renal injuries: management guidelines from a 25-year experience. J Urol 172: 687-690. 
12. McCombie SP, Thyer I, Corcoran NM, Rowling C, Dyer J, et al. (2014) The conservative management of renal trauma: a literature review and practical clinical guideline from Australia and New Zealand. BJU Int 114: 13-21.

13. Buckley JC, McAninch JW (2006) The diagnosis, management, and outcomes of pediatric renal injuries. Urol Clin North Am 33: 33-40.

14. McClung CD, Hotaling JM, Wang J, Wessells H, Voelzke BB (2013) Contemporary trends in the immediate surgical management of renal trauma using a national database. J Trauma Acute Care Surg 75: 602-606.

15. Umbreit EC, Routh JC, Husmann DA (2009) Nonoperative management of nonvascular grade IV blunt renal trauma in children: meta-analysis and systematic review. Urology 74: 579-582.

16. Prasad NH, Devraj R, Chandriah GR, Sagar SV, Reddy ChR, et al. (2014) Predictors of nephrectomy in high grade blunt renal trauma patients treated primarily with conservative intent. Indian J Urol 30: 158-160.

17. McGuire J, Bultitude MF, Davis P, Koukounaras J, Royce PL, et al. (2011) Predictors of outcome for blunt high grade renal injury treated with conservative intent. J Urol 185: 187-191.

18. Somani BK, Nabi G, Thorpe P, Hussey J, McClinton S (2006) Therapeutic transarterial embolisation in the management of benign and malignant renal conditions. Surgeon 4: 348-352.

19. Tait CD, Somani BK (2012) Renal trauma: case reports and overview. Case Rep Urol 2012: 207872.

20. Somani BK, Nabi G, Thorpe P, McClinton S (2006) Endovascular control of haemorrhagic urological emergencies: an observational study. BMC Urol 6: 27.
21. Saour M, Charbit J, Millet I, Monnin V, Taourel P, et al. (2014) Effect of renal angioembolization on post-traumatic acute kidney injury after high-grade renal trauma: a comparative study of 52 consecutive cases. Injury 45: 894-901.

22. Bookstein JJ, Ernst CB (1973) Vasodilatory and vasoconstrictive pharmacoangiographic manipulation of renal collateral flow. Radiology 108: 55-59.

23. Glass AS, Appa AA, Kenfield SA, Bagga HS, Blaschko SD, et al. (2014) Selective angioembolization for traumatic renal injuries: a survey on clinician practice. World J Urol 32: 821-827.

24. Hotaling JM, Sorensen MD, Smith TG 3rd, Rivara FP, Wessells $H$, et al. (2011) Analysis of diagnostic angiography and angioembolization in the acute management of renal trauma using a national data set. J Urol 185: 13161320.

25. Hotaling JM, Wang J, Sorensen MD, Rivara FP, Gore JL, et al. (2012) A national study of trauma level designation and renal trauma outcomes. J Urol 187: $536-541$

26. McGuire J, Bultitude MF, Davis P, Koukounaras J, Royce PL, et al. (2011) Predictors of outcome for blunt high grade renal injury treated with conservative intent. J Urol 185: 187-191.

27. Heller MT, Schnor N (2014) MDCT of renal trauma: correlation to AAST organ injury scale. Clin Imaging 38: 410-417.

28. Long JA, Fiard G, Descotes JL, Arnoux V, Arvin-Berod A, et al. (2012) Highgrade renal injury: non-operative management of urinary extravasation and prediction of long-term outcomes. BJU Int 111: 249-255.

29. Tasian GE, Aaronson DS, McAninch JW (2010) Evaluation of renal function after major renal injury: correlation with the American Association for the Surgery of Trauma Injury Scale. J Urol 183: 196-200. 